Patricio De Navascués

\title{
O crucifer bone lucisator (Prudence, cath. 3.1)
}

\author{
Doctrine ancienne en termes nouveaux
}

\section{Sur le Cathemerinon}

Sur le style et la pensée de Prudence, un grand nombre de pages ont été écrites au cours de ces dernières décennies. Les questions les plus diversifiées, de grande envergure et incidence, ont été suscitées concernant l'œuvre de Prudence: je pense aux problèmes textuels qu'en présente la tradition manuscrite, aux questions littéraires touchant la métrique -plus d'une fois- toute originelle, à la chronologie et signification exacte de sa Préface, à la considération de son œuvre intégrale en tant qu'un seul poème, etcetera. Une bonne exposition sur les principales opinions soutenues par les specialistes jusqu'en 1993 a été donné par A. A. R. Bastiaensen dans le volume Early Christian Poetry. ${ }^{1}$ Après cette date, les chercheurs n'ont pas manqué de produire de nouvelles éditions, traductions, articles, etcetera. À titre illustratif, nous pourrions souligner les trois volumes Prudentiana, confectionnés par un expert de Prudence, de grande renommée, le professeur, déjà émérite à l'Université de Münster, Christian Gnilka. ${ }^{2}$

Les poèmes de Prudence ont provoqué dans la recherche moderne, des réactions contrastées. Aujourd'hui ils continuent à le faire. Toutefois, il y a certains points qui méritent l'assentiment de presque tous les spécialistes de Prudence. En tenant compte, ou mieux en considérant les paroles mêmes de Bastiaensen, la poésie de Prudence présente 'a strong internal unity', et de façon toute particulière, il s'agit d'une cohérence au niveau de la pensée théologique même, et mieux encore, d'une cohérence plus forte qui en fait une unité d'inspiration: Prudentius peut par conséquent, être considéré comme un écrivain, qui est fortement et hautement convaincu que la foi chrétienne est vraie et digne d'être honorée au moyen de la poésie.

Ce que je propose ici n'est qu'une approche beaucoup plus modeste. Ce n'est pas une réflexion générale sur un aspect particulier de l'œuvre du poète Calagurritanus. Par contre, je me suis concentré sur un verset de son Cathemerinon, je dirais plutôt, sur un seul mot. En effet, au cours de mes recherches sur Prudence, je me suis pris d'enthousiasme pour le début d'un de ses deux hymnes dédiés au repas. Il s'agit du Hymnus ante cibum, le troisième du Cathemerinon.

Le passage examiné appartient donc à cette collection d'hymnes appelée $\mathrm{Ca}$ themerinon, dans laquelle le poète hispanique a essayé de fournir une méditation

1 Cf. Bastiaensen (1993) 101-134.

2 Cf. Gnilka (2000. 2001. 2003). 
rythmée pour chaque moment de la journée (c'est d'ailleurs pour ça qu'il s'appelle quotidienne, cathemerinon) et de l'année. Il n'est pas inutile de rappeler que l'œuvre du Cathemerinon comprend douze hymnes, disposés en six paires en forme de chiasme. Ce qui nous donne donc la répartition suivante: ${ }^{3}$

\begin{tabular}{ll}
\hline Jour & Année \\
\hline I-II: Matin & XI-XII Noël et Epiphanie \\
\hline III-IV: Repas & IX-X: Pâque \\
\hline V-VI: Soir & VII-VIII: Carême \\
\hline
\end{tabular}

\section{Sur l'hymne ante cibum}

Notre verset appartient alors aux couples centraux, qui sont consacrés à la nourriture. Pour le cycle du jour, deux hymnes (troisième et quatrième) avant et après le repas; pour le cycle de l'année, deux hymnes (neuvième et dixième) autour de la Pâque. Ce n'est pas un lien superficiel. Par contre, chez un auteur comme Prudence, cela nous montre la perspective avec laquelle nous devrions lire l'hymne avant le repas: avec une perspective christologique, et même plus, pascale.

L'hymne en question a été commenté plusieurs fois. Il s'agit d'un hymne de quarante-et-une strophes pentastiques, deux cent cinq versets, composé pour être chanté avant le repas, afin d'aider les hommes à manger en découvrant le goût de Dieu (fercula nostra deum sapiant, cath. 3.16), la grâce du Christ dans les coupes et sur les plats (cf. cath. 3.16 ; 11-15) et par ailleurs pour que nos occupations sérieuses, nos récréations, nos paroles, nos jeux, en un mot nos personnes et nos actions (cath. 3,16-20) soient conduits par la Sainte Trinité (cf. cath. 3.20). La perspective, à mon avis, est très haute. Prudence projette sur le modeste thème du repas, une lumière, qui met en relief plusieurs aspects implicites (christologiques, anthropologiques, sotériologiques) dans l'acte de se nourrir.

Le mètre utilisé c'est le trimètre dactylique hipercatalectique, ou autrement dit, le tetramètre dactylique catalectique in syllabam (- U U - U U - U U x), exactement comme il l'a fait pour le troisième hymne du Peristephanon, dédié à Sainte Eulalie et en symétrie avec le dixième hymne sur la Pâque, qui présente des strophes dimètre anapestique catalectique (la symétrie émerge en considérant l'anapeste comme un dactyle inverti). ${ }^{4}$ En outre, en contraste avec les deux premiers hymnes qui consti-

3 Cf. Fontaine (1981) 184: "Au centre de chacune des deux moitiés, une attention particulière est accordée à la spiritualité des repas et des jeûnes. Autour de ces deux centres, se répondent en chiasme: dans le jour, le passage de la lumière à la nuit, et dans l'année, celui (inverse) de la mort à la résurrection».

4 Cf. Bergman (1921) 55-67. 
tuent le Cathemerinon, qui ont été faits en strophes strictement ambrosiennes, dimètres ïambiques, le mètre choisi ici par Prudence se place dans un niveau plus haut et, pour cette occasion, il dénonce l'influence d'Ausone. ${ }^{5}$

Si nous pensons au public auquel notre auteur s'est adressé, nous pouvons conclure, en empruntant les paroles à J. Fontaine, que «ce n'est pas le public mondain des recitationes romaines... pas davantage l'assemblée liturgique, pour laquelle écrivaient d'abord les évêques hymnodes... La communauté, rassemblée ou dispersée, pour laquelle Prudence a écrit ses poèmes, mène un style de vie comparable, dans ses aspects matériels et moraux, à celui de ces autres propriétaires terriens qu'ont été Sulpice en Aquitaine, Paulin en Campanie, ou l'ancien préfet Dardanus dans sa 'Théopolis' des Basses-Alpes. Le lyrisme de Prudence semble d'abord destiné à cette catégorie d'aristocrates lettrés, retirés sur leurs terres pour y mener une vie d'ascétisme monastique». ${ }^{6}$

Avant de mettre un point final à cette petite introduction, il faudra encore mentionner qu'ont été publiés heureusement au cours de ces dernières années, deux commentaires sur notre poème. L'un d'entre eux, le plus récent, a été écrit par le professeur émérite Gerard O’Daly en 2012;'7 l'autre c'est une œuvre de Maria Becker (c'est-à-dire, dans la tradition de Christian Gnilka), professeure à la Wilhelm Winter Universität, en Münster, publié il y a déjà un peu plus de dix ans, en 2006. Dans ce cas, l'œuvre concerne exclusivement le troisième hymne. ${ }^{8}$

En ce qui concerne la structure de l'Hymnus ante cibum, Maria Becker a réfuté la thèse de Jean Louis Charlet, ${ }^{9}$ selon laquelle, nous sommes en face d'une structure composée de cinq parties, qui renferme au centre - en imitant le rôle central joué par les exempla utilisés par Horace et Pindare- le récit biblique, le récit d'Adam et Eve au paradis, en en soulignant l'importance. Par contre, Maria Becker ${ }^{10}$ a défendu une structure à quatre parties, de type binaire, qui semble être acceptée aussi par Gerard O'Daly, ${ }^{11}$ et divise en deux la thématique principale de cet hymne: d'une part, la louange envers les dons de la Création; d'autre part, le récit de l'histoire de l'homme, en mettant en évidence Adam, Ėve, le péché, le nouvel Adam (Christ), né de la vierge Marie. Récement, dans sa thèse defendue à l'Université de Toronto, Cillian Conor O’Hogan soutiens que le text biblique de la Genèse (sur la création et le péché) se trouve dés le début de l'hymne en lui offrent d'une structure. ${ }^{12}$

5 Cf. Charlet (1980) 96-98.

6 Fontaine (1981) 181.

7 Cf. O’Daly (2012).

8 Cf. Becker (2006).

9 Cf. Charlet (1982) 61-72.

10 Cf. Becker (2006) 23-28.

11 Cf. O’Daly (2012) 96-97.

12 Cf. O’Hogan (2012) 126-130. 


\section{Sur cath. 3.1-5}

Du point de vue textuel, il n'y a pas de problème en ce qui concerne les cinq premiers versets de l'hymne ante cibum. Voici le début, la première strophe, de grande classe:

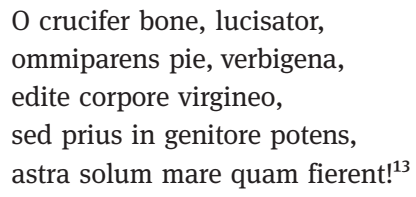

Ce début constitue un bon exemple pour illustrer l'un des traits distinctifs de Prudence, c'est-à-dire, sa capacité pour l'innovation lexicale. En l'espace de deux versets nous avons trouvé déjà jusqu'à trois mots forgés par lui: crucifer, lucisator, verbigena. Selon Becker, ${ }^{14}$ Prudence a essayé d'éviter, contrairement à l'usage qu'en fait Horace, la substitution du mètre dactylique par le mètre spondéen, en cherchant ainsi le principe de l'isosyllabismus, déjà trouvé dans la poésie ambrosienne. Cela a provoqué aussi un haut style avec beaucoup de mots (souvent néologismes) comprenant quatre ou cinq syllabes. Il y en a beaucoup tout au long du poème, mais seulement ici, dans les deux premiers versets, nous en avons trouvé trois: lucisator, omniparens, verbigena.

Il ne fait aucun doute que ce commencement nous offre la base christologique du poème entier. Le début avec l'interjection 'o' et cette accumulation aussi grande de mots inhabituels utilisés comme invocations toutes orientées vers le Christ, n’a pas d'équivalents dans les autres débuts que nous trouvons dans les hymnes du Cathemerinon. Nous sommes en présence de ce qu'on appelle «le mélange des genres dans la poésie de Prudence», plus précisément ici, nous constatons la présence de noms caractéristiques du style épique (lucisator, omniparens, verbigena) à l'intérieur d'un poème lyrique. ${ }^{15}$

Nous pouvons aussi remarquer la structure parallèle constatable entre les deux premiers versets: ils se répartissent en deux cola: le premier colon dans les deux versets avec six syllabes ( $O$ crucifer bone et omniparens pie); le deuxième, avec quatre

13 Cf. Lavarenne (1955) 12. Et voici la traduction offerte par l'édition française de M. Lavarenne en Prudence (1955) 12: «O porte-croix plein de bonté, créateur de la lumière, saint producteur de toutes choses, engendré par le Verbe et né du corps d'une vierge, mais déjà puissant dans le Père avant que ne fussent faits les astres, le sol et la mer!»; voici la version anglaise de O’Daly (2012) 83: «O kind cross-carrier, light-sower, / caring All-father, Word-born, / brought forth from a virgin's body, / but powerful in the Father / before stars, earth, sea were made». Et voici l'allemande de Becker (2006) 31: O guter Kreuzträger, Lichtbringer, / gütiger Allvater, durch das Wort Geborener, / dem Körper der Jungfrau entsprossen, / aber zuvor schon im Vater mächtig, / bevor Sterne, Erde und Meer geschaffen wurden!

14 Cf. Becker (2006) 22.

15 Cf. Fontaine (1975) 763; Lühken (2002) 203-207. 
syllabes (lucisator et verbigena). ${ }^{16}$ Par ailleurs, il a été aussi mis en évidence la gradatio où Prudence a disposé les quatre appellatifs du Christ: crucifer, lucisator, omniparens, verbigena, en passant des substantifs aux participes. ${ }^{17}$

Mais si nous considérons maintenant plutôt la signification de cette grande ouverture, qu'observons-nous?

On pourrait dire que la dénomination de crucifer doit être mise en rapport, à la manière de chiasme, avec verbigena, parce que tous les deux correspondent au Christ en tant que Sauveur: crucifer nous renvoie au mystère de la Rédemption et à la Pâque; verbigena, à son tour, au mystère de l'Incarnation. En tout cas, nous restons dans l'œuvre du salut. D'autre part, nous remarquons que lucisator et omniparens, qui nous indiquent l'œuvre de la Création, louent le Christ en tant que Créateur. C'est la thèse de Becker. ${ }^{18}$

À son tour, le premier mot, crucifer, 'celui qui porte la croix', nous offre, dès le début, le motif de la croix et correspond à la fin de l'hymne suivant dédié aussi au repas et qui s'appelle hymnus post cibum. Là, en effet, on fait une référence aux chrétiens qui doivent porter la croix (cf. cath. 4.102). La croix embrasse l'acte de nutrition. C'est la référence pour se nourrir. On peut donc affirmer que, dans l'intention de Prudence, existe la volonté de faire -grâce à la croix- un lien entre la louange envers Dieu et l'exigence étique concernant la nutrition. ${ }^{19}$ De plus ici, en ouvrant solennellement le poème, avec cette invocation, on satisfait à la fonction d'identifier sans ambigüité le Dieu, que le poète -ou les hommes- prient.

En outre, à la fin du verset, nous nous retrouvons en face d'un autre néologisme de Prudence: lucisator, ${ }^{20}$ c'est-à-dire, 'semeur de la lumière'. Je pense qu'il faut maintenir dans les versions modernes la traduction 'semeur', sans vouloir obtenir une autre traduction un peu plus figurée, comme 'créateur de la lumière' ou 'Lichtbringer'. En effet, l'image des semis, de la plantation, est capital dans le contexte d'un hymne dédié précisément à l'acte de la nutrition fait à partir des fruits de la terre.

Avec ce mot, Prudence, d'une part, baptise la tradition païenne habituée à s'adresser à Sator, c'est-à-dire à Jupiter; et d'autre part, il se sert de l'image de la lumière, si riche de significations pour un chrétien (et pour Prudence même): ${ }^{21}$ la lumière de la Création, la lumière de la grâce baptismale, la lumière de la gloire céleste, etcetera.

16 Cf. Becker (2006) 49.

17 Cf. Becker (2006) 49.

18 Cf. Becker (2006) 49.

19 Cf. Becker (2006) 50.

20 Dans l'ensemble de l'oeuvre de Prudentius, on peut trouver cent trente quatre mots avec le suffix -tor, dont il y a huit, qui sont néologismes: emancipator, infusor, lancinator, lucisator, perdomitor, traiector, uerberator, unicultor, cf. Mariner Bigorra (1959) 199-236.

21 Cf. Gosserez (2001). 
Enfin, le petit bone qui suit crucifer fait pendant aussi au pie, qui accompagne omniparens, dans le deuxième verset, et, selon Becker, tous deux (bone et pie) soulignent l'aspect sotériologique propter nos.

Apparemment tout semble être clair. Mais O’Daly indique justement que cette référence à la croix -Crucifer- ne trouve aucune mention explicite dans le reste du poème ante cibum. Par ailleurs, ce que Becker a proposé pour le comprendre, en faisant un chiasme avec verbigena ne s'avère pas conclusif sur ce point.

À mon avis, afin de mieux comprendre cette image du Christ portant la croix, crucifer, un peu énigmatique, tant du point de vue littéraire (un neologism qui, en outre, est presque un hapax) que théologique, il faut se rappeler de la tradition chrétienne ancienne, antérieure à Prudence, forgée par la mentalité des chrétiens prenicéens, qui étaient capables de découvrir la présence de la croix derrière une multitude d'éléments. Là, je pense notamment à l'image de la charrue, du soc, de l'aratrum.

\section{Crux - aratrum}

La réflexion sur la croix, aux premiers siècles du christianisme, déborde très souvent le cadre historique qui se limite à la crucifixion du Nazaréen au point d'en faire une clé capable de contenir l'histoire entière du salut, racontée dès le début dans le livre de la Genèse jusqu'à son exposé final dans l'Apocalypse de Jean. En effet, cette tradition, qui a peut-être été développée à partir d'un verset du prophète Esaïe 2,3, selon lequel, dans les temps messianiques, les épées se changeront en charrues, c'est-à-dire, qu'il n'y aura plus aucune guerre, et que par contre, les hommes se consacreront à la culture de la terre. C'est déjà Justin, qui a mis en rapport la charrue, le soc, avec la croix, mais nous allons nous appesantir sur Irénée de Lyon, qui nous a laissé de très intéressantes lignes à propos de l'équivalence entre la croix et l'aratrum. Voici le texte d'Irénée ( $a d v$. haer. IV, 34, 4):

Hic autem est Dominus noster, et in hoc est sermo verus, quoniam ipse est qui aratrum fecit et falcem intulit, hoc est hominis primam seminationem quae fuit secundum Adam plasmatio, et in novissimis temporibus per Verbum collectam fructificationem.

«Or, c'est notre Seigneur et »en lui se vérifie la parole« (Jn 4.37), car c'est lui qui a fait la charrue et qui a apporté la faucille, ce qui signifie, d'une part, le premier ensemencement de l'homme que fut son modelage en Adam et, d'autre part, la récolte du fruit faite par l'entremise du Verbe dans les derniers temps ${ }^{22}$

22 Cf. Rousseau (1965) 858-859. 
La parole vérifiée (cf. in hoc est sermo verus) c'est celle qui dit: l'un sème, l'autre moissonne (Jn 4.37). ${ }^{23}$ Pour les gnostiques valentiniens, ce lieu biblique fournit la base pour distinguer entre la seminatio réalisée par le Démiurge psychique (l'un sème) et la collecta, la moisson de l'homme menée par le Sauveur pneumatique (l'autre moissonne) en parfaite correspondance avec la division entre l'Ancien Testament (régime d'ignorance sous le gouvernement de Yahvé, le Dieu inferieur) et le Nouveau Testament (régime de connaissance pour les gnostiques, sous la providence du Dieu supérieur).

Afin de mieux contraster cela, Irénée de Lyon mettra un accent particulier sur l'identité entre le Semeur (Christ en tant que Demiurge) et le Moissonneur (Christ en tant que Sauveur). ${ }^{24}$

La suite du texte d'Irénée se présente comme suit ( $a d v$. haer. IV.34.4):

\begin{abstract}
Et propter hoc quod initium fini conjungebat, et utrorumque Dominus exsistens, in fine quidem aratrum ostendit, lignum copulatum ferro, et sic ejus expurgavit terram, quoniam firmum Verbum adunitum carni et habitu tali confixus emundavit silvestrem terram; initio autem falcem figurabat per Abel, significans justi generis hominum collectionem: Vide enim, inquit, quomodo justus perit, et nemo intuetur, et viri justi tolluntur, et nemo excipit corde (Is 57.1). Haec autem in Abel quidem praemeditabantur, in prophetis vero praeconabantur, in Domino autem perficiebantur, et in nobis autem idipsum, consequent corpore suum caput.

«Et c'est pourquoi, comme il unissait le commencement à la fin, étant le Seigneur de l'un et de l'autre, d'une part, à la fin il montra la charrue, c'est-à-dire le bois uni au fer et nettoyant ainsi la terre; car le Verbe solide, en étant uni à la chair et en étant fixé à elle de cette manière, a nettoyé la terre embroussaillée; d'autre part, dès le commencement, il préfigurait la faucille par Abel, signifiant par là la récolte de la race juste des hommes. 'Car vois, est-il-dit (Is 57.1), comment le juste a péri, et nul ne le remarque, comment les hommes justes sont supprimés, et nul ne le saisit en son coeur'. Cela était inauguré en Abel, puis proclamé par les prophètes, puis accompli dans le Seigneur, et il en va encore de même pour nous, le corps suivant sa tête. $»^{25}$
\end{abstract}

Dans l'Ancient Testament prophétique, le Seigneur fit la charrue, autant qu'il fit l'homme, Adam, destiné à son tour à semer le fils de Dieu dans la création. Dans le Nouveau Testament -apostolique- le Seigner a montré la charrue, en tant qu'il a été crucifié, en produisant ainsi comme conséquence la purification de la terre embroussaillée par le péché d'Adam et l'ensemencement définitif, afin que tous les hommes deviennent fils de Dieu. Dans la croix se trouvent réunis le bois, c'est-à-dire la faiblesse de la nature humaine, et le fer, c'est-à-dire, la nature divine, lumineuse, du Fils de Dieu. «Le Verbe incarné et cloué sur la croix purifia cette terre inutile et

23 Le text grec dit: દ̉v yò $\theta \varepsilon \rho i \zeta \omega v$.

24 Cf. Orbe (2012) 1146: «Un seul et le même, le Sauveur, a fait la charrue, outil d'ensemencement, pour semer avec Adam le genre humain dans le monde; il a aussi introduit la faucille, outil de moisson, pour récolter dans les derniers temps le genre humain et le rassembler dans les greniers du Père».

25 Cf. Rousseau (1965) 858-861. 
inféconde pour son Seigneur en la rendant utile et féconde». ${ }^{26}$ Ainsi le Seigneur, dans les derniers temps, montra l'aratrum, en tant que porteur de la croix, outil d'ensemencement et purification (au début avec Adam et dans les derniers temps grâce au mystère de la croix). ${ }^{27}$

Nous pouvons donc constater comment l'image de la charrue avec le soc, contemplée dans une perspective christologique, est capable de suggérer l'histoire entière du salut.

Le lien entre Christ et l'aratrum appartenait à l'univers d'idées que relayaient les chrétiens. Une preuve en est l'apparition d'aratrum, en tant que titre christologique, à l'intérieur d'une liste de noms du Christ dans les Actes de Pierre avec Simone 20 et dans les Actes de Jean 109.

Mais nous pouvons nous rapprocher davantage de Prudence, en rappelant un morceau du Sermo 38 de saint Maxime de Turin, De cruce et resurrectione Domini, oú nous pouvons lire (Sermo 38. 2. 3. 4):

\footnotetext{
Grande ergo crucis est sacramentum... Sed et bonus agricola cum parat terrae solum vertere et vitae alimenta perquirere, nonnisi per signum crucis id facere conatur. Dum enim aratro dentalem subicit adfigit aures stevam inserit, figuram crucis imitatur; conpositio enim ipsa similitudo quaedam est dominicae passionis... Nam ex quo homo dominus Iesus, qui ipsam crucem gestabat, sepultus in terra est, veluti disrupta ab eo et exarata terra omnes quos retinebat mortuos germinavit. ${ }^{28}$

«Donc il est grand le mystère de la croix... Et aussi le bon paysan, lorsqu'il se prépare pour retourner le terrain et chercher la nourriture pour la vie, il ne le fait autrement sinon au travers du signe de la croix. En effet, lorsqu'il met le dental sous la charrue, fixe les (aureilles) et insère le manche, imite la figure de la croix. En effet, la composition même présente une certaine ressemblance à la passion du Seigneur... En effet, à partir du moment où l'homme Jésus, le Seigneur, qui portait la croix même, fut enseveli dans la terre, comme brisée et labourée par Lui, il fit germer tous ceux que [la terre] retenait morts».
}

Nous retrouvons à nouveau le symbole de l'aratrum, avec lequel le bonus agricola travaille la terre. Dans la transposition au contenu chrétien, alors le bonus agricola est devenu Jésus, qui ipsam crucem -la charrue- gestabat, et qui, par sa mort et sépulture, devint capable de travailler la terre, c'est-à-dire, l'homme, qui était mort, pour lui redonner la vie. Le motif agricole de la charrue est devenu très spontanément pascal.

Maintenant aussi pouvons-nous nous intéresser, du point de vue géographique, à la patrie de Prudence et découvrir dans un sermon, cette fois-ci, appartenant à Grégoire d'Elvire, les paroles suivantes, cf. Hom. VI.19:

26 Cf. Orbe (2012) 1148.

27 Cf. Orbe (1994) 571-579; Doignon (1955) 535-544.

28 Cf. Mutzenbecher (1962) 149-150. 
Quem ideo taurum propheticus spiritus appelavit, eo quod primum aratrum crucis suae patibulo ipse portaverit, unde dura gentium pectora subacta necessario semini, id est Sancto Spiritui praeparaverit... ${ }^{29}$

"C'est pour ça que l'esprit prophétique l'appela bœuf, parce que Lui-même porta à l'échafaud la charrue de sa croix, d'où, une fois labourés les cœurs des Gentils qui étaient endurcis, prépara pour eux convenablement la semence, c'est-à-dire, l'Esprit Saint.»

\section{Vers une conclusion}

Dans les textes que l'on vient de citer (Irénée, Maxime, Grégoire) nous avons pu vérifier la facilité avec laquelle les premiers chrétiens mettaient en rapport la charrue avec la croix. En outre, nous avons trouvé chez Maxime et Grégoire l'image du Christ en portant de l'aratrum de sa croix. Et encore plus, chez Maxime nous avons trouvé à côté d'agricola, l'adjectif bonus, c'èst-à-dire le même que Prudence a mis à côté pour crucifer: o crucifer bone. En ce qui concerne la littérature païenne, nous connaissons le topos du bonus agricola, construi déjà à partir de Caton. ${ }^{30}$ Ce que nous remarquons chez Maxime et, à mon avis, aussi chez Prudence, concernant le topos du bon paysan, c'est le passage du module païen au module chrétien à travers l'équivalence crux=aratrum, où apparaît le Christ comme le bonus agricola.

Par ailleurs, point n'est besoin de vous rappeler l'importance de Virgile chez Prudence. Du reste, notre aratrum apparaît mystérieusement au début même des Georgiques, lorsque Virgile invoque le uncique puer monstrator aratri (G. I.19). À propos de ce lieu virgilien Servius, commentateur de Virgile, qui a vécu à l'époque de Prudence, a dit: bene autem tacuit de nomine et generaliter ait 'puer'. ${ }^{31}$ Cet enfant..., était-il pour Prudence le Christ? Et peu après, Virgile cite à nouveau le soc et la charrue parmi les outils avec lesquels le paysan travaille. Elle occupe la première place (cf. G. I.160 -162: dicendum est quae sint duris agrestibus arma, / quis sine nec potuere seri nec surgere messes: / vomis et inflexi primum grave robur aratri).

Dans cette perspective, si notre hypothèse est admise, le point de départ christologique de l'hymnus ante cibum acquiert de nouveaux profils. O crucifer bone lucisator... autant dire o bon porteur de la charrue mystique, o bone agricola, afin de rappeler, non seulement la fonction de Christ Sauveur, mais déjà aussi, au début de la Création, la fonction de Christ, qui prépare la terre d'Adam et qui prépare, en tant que Verbe Créateur, en général, la terre, qui donne des fruits pour les hommes.

$O$ crucifer a probablement des résonances cosmiques, liées au theologoumenon de la croix cosmique, si spontanée pour les chrétiens des premiers siècles, et c'est pour ça que ce début est capable de susciter avec un seul mot plusieurs connota-

29 Cf. Pascual Torró (1997) 166.

30 Cf. Stone (1998) 103-113.

31 Servii, Comm. in Verg. Georg. I, 19. 
tions: le travail du Verbe qui prépare la terre, qui doit soutenir les hommes; le travail du Verbe, au début de l'histoire, sur la terre, qui est l'homme, pour y ensemencer la lumière de l'esprit; le travail du Verbe, mais maintenant déjà incarné, pour purifier la terre de toute iniquité -c'est-à-dire-, pour purifier la chair des hommes, devenue sauvage à partir du péché, devenue morte, afin de semer en elle, la puissance lumineuse de l'Esprit vivifiant.

Il y a donc deux types de plantations: l'ensemencement de la terre qui donne les fruits (pour manger, je rappelle que nous nous trouvons dans l'Hymnus ante cibum) et l'ensemencement de l'homme appelé à devenir fils de Dieu. Tous les deux ont commencé, grâce au Christ, dès le début de la Création. Il y a un seul Semeur et il y a, en somme, un unique récepteur des deux semences, qui est le corps humain, la chair. Elle -la chair- reçoit soit les fruits de la terre, soit la lumière de l'Esprit. En fait, il n'y a qu'un unique but poursuivi par le Semeur: le corps virginal né de la Vierge, le vrai fruit de la création, que nous pouvons lire dans la troisième ligne de notre strophe: edite corpore virgineo. Si nous apprenons à manger et à boire, selon la volonté du bon Semeur, Prudence semble dire que nous aurons la même espérance qui fut réservée au corps virginal: Spes eadem mea membra manet (cath. 3, 201) et toute la création convergera finalement vers l'objectif principal: la gloire lumineuse de la chair des hommes nourries par la Création labourée avec la charrue et ressuscités par le Verb au moyen de la croix.

Le rythme initial devient vraiment intense: $O$ crucifer bone, qui avec son soc prépare la terre de la Création et la chair d'Adam, et qui peu après retourne le terrain et l'homme; lucisator, qui sème la lumière (l'esprit saint) pour les féconder au debut de la Création qua deus et dans la Pâque à travers son esprit qua deus et homo deificatus; omniparens pie, qui fait naître toutes choses au debut et dans la Pâque; verbigena edite corpore virgineo, qui par le Verbe est né de la Vierge...

\section{Bibliographie}

Bastiaensen (1993): Antoon A. R. Bastiaensen, «Prudentius in recent literary criticism», in: Jan den Boeft and Anton Hilhorst (eds.), Early Christian Poetry, Leiden, 101-134.

Becker (2006): Maria Becker, Kommentar zum Tischgebet des Prudentius (cath. 3), Heidelberg. Bergman (1921): Johan Bergman, Aurelius Prudentius Clemens, der grösste christliche Dichter des Altertums, Dorpat.

Charlet (1980): Jean-Louis Charlet, L'influence d'Ausone sur la poésie de Prudence, Paris. Charlet (1982): Jean-Louis Charlet, La création poétique dans le Cathemerinon de Prudence, Paris. Doignon (1955): Jean Doignon, «La salut par le fer et le bois. Notes de philologie et d'éxègese sur Adversus Haereses IV, 34, 4», in: Recherches de Science Religieuse 43, 535-544.

Fontaine (1975): Jacques Fontaine, "Le mélange des genres dans la poésie de Prudence», in: Forma Futuri. Studi in onore di M. Pellegrino, Torino, 755-777.

Fontaine (1981): Jacques Fontaine, Naissance de la poésie dans l'Occident chrétien. Esquisse d'une histoire de la poésie latine chrétienne du Ille au Vle siècle, Paris.

Gnilka (2000. 2001. 2003): Christian Gnilka, Prudentiana I. Crítica. II. Exegetica. III. Supplementum, München/Leipzig. 
Gosserez (2001): Laurence Gosserez, Poésie de lumière. Une lecture de Prudence, Louvain. Lavarenne (1955): Maurice Lavarenne (ed.), Prudence Tome I. Cathemerino Liber (Livre d'heres), Paris.

Lühken (2002): Maria Lühken, Christianorum Maro et Flaccus. Zur Virgil- und Horazrezeption des Prudentius, Göttingen.

Mariner Bigorra (1959): Sebastián Mariner Bigorra, «El latín de la península: léxico», in: Enciclopedia Lingüística Hispánica I, CSIC, Madrid, 199-236.

Mutzenbecher (1962): Almut Mutzenbecher (ed.), Maximi episcopi Taurinensis Collectio sermonum antiqua, Turnholti.

O’Daly (2012): Gerald O’Daly, Days linked by song. Prudentius' Cathemerinon, Oxford.

O'Hogan (2012): Cillian Conor O'Hogan, Geography and Space in the Poetry of Prudentius, diss. University of Toronto.

Orbe (1994): Antonio Orbe, Estudios sobre la teología cristiana primitiva, Madrid.

Orbe (2012): Antonio Orbe, Introduction à la théologie des II et III siècles, vol. II, Paris.

Pascual Torró (1997): Joaquín Pascual Torró (ed.), Gregorio de Elvira. Tratados sobre los libros de las Santas Escrituras, Madrid.

Rousseau (1965): Adelin Rousseau (ed.), Irénée de Lyon. Contre les hérésies. Livre IV, Paris.

Stone (1998): David L. Stone, «Culture and Investment in the rural Landscape. The Nort African bonus agricola», in: Antiquités Africaines 34, 103-113. 
